\title{
An Automatic Method to detect defects for Solid Rocket Motor FU Qian ${ }^{1,}$, ${ }^{\text {ZHAO Jiuling }}{ }^{1}$ \\ ${ }^{1}$ School of the Second Artillery Engineering University, Xi'an 710025, China. \\ afuqianyanfen2009@163.com
}

Keywords: solid rocket motor; bot-hat transformation; threshold segmentation.

\begin{abstract}
Since the traditional defects detection methods on the CT image was not highly automated and could not lead to satisfied effects, a new defects detection method of the solid rocket motor was proposed based on the bot-hat transformation and threshold segmentation. Firstly, the bot-hat transformation was adopted to transform original images to gray images with enhanced valley values; secondly, an automatic threshold segmentation algorithm was designed to segment the defects in the gray images. It was found that with the new proposed defects detection method, the spatial distributions, outlines and size characteristics of the defects could be clearly detected, which demonstrated an effective detection result.
\end{abstract}

\section{Introduction}

The solid rocket motor (SRM) is main part of the solid rocket. In the life cycle of SRM, such as manufacture, storage, testing, launching, etc., there would be unvoided defects in SRM, including debonding, cracks, porosity, etc [1]. If there are potential safety problems in the device, the defense and the economy will be damaged seriously. Therefore, it is of great importance to detect the defects of SRM and extract the defects.

Industrial CT technology has the characteristics of intuitionistic imaging, high resolution, not being restricted by the geometric construction of the workpiece [2], which led to the wide use in nondestructive testing (NDT) and nondestructive evaluation (NDE). It is recognized as the best nondestructive detection methods by international nondestructive field. However, the CT image is the fault image of the workpiece, and the judgment of the defects still needs the interpretation of the professional technicians, and there is a certain fluctuation. How to improve the automatic and intelligent degree of defect detection is an urgent problem to be solved [4]

Image segmentation is the key technology for the detection of the defects of the SRM CT images. The typical significance of image segmentation is how to extract the consistency region and the interested object in the process of image processing [4]. The accuracy of segmentation determines the final success of the computerized analysis [5]. At present, there is a thousand kinds of methods of image segmentation, which are mainly divided into region based, edge based and new image segmentation algorithm based on new theory. However, there is no segmentation method can be used in any image. Therefore, it can only be proposed based on different application background. Aiming at the flaw detection of SRM, an improved watershed algorithm is proposed in [6]. However, the algorithm still has an over-segmentation problem. Moreover, the algorithm is targeted at the typical sea missile CT images. If the algorithm is applied to the general solid engine CT images, over-segmentation phenomenon will be more significant. Zhu Min et al. [7] proposed an automatic segmentation method with the integration of edge detection, mathematical morphology and multi segmentation. Because the gray value of core hole, defect and column is cross each other in the real images, the method has its limitation applied to detect real defects.

In this paper, a new method with the integration of the bot-hat transformation and threshold segmentation algorithm is proposed, considering the gray distribution of SRM CT images. 


\section{Defect Detection Scheme.}

The SRM is mainly composed of shell, insulation layer, artificial unsticking layer, lining layer, propellant and core hole, etc. [8]. Among them, the shell, the insulation layer and the lining layer is very thin, and the artificial unsticking layer contains air. Therefore, observing the CT image of SRM by the naked eye, it can be divided into the outer structure (including the shell and the insulation layer), the artificial unsticking layer, the propellant (fused the lining layer). The outer layer and the propellant are relatively high gray value structures, while the artificial unsticking layer and the core hole are relatively low, which are close to the background gray value. In this paper, the defects of SRM are mainly distributed in the outer structure and the structure of the propellant, such as porosity, debonding and crack. Because the flaws are small and filled with air, their gray values and pixel distribution have their unique features. In this paper, an SRM defect detection scheme is proposed for these features.

\subsection{Preprocessing of removing the aperture for the CT image}

Fig. 1 (a) is a SRM CT image with a defect with a distance of $120 \mathrm{~cm}$ to rear joint end, and the size is $2048 * 2048$ pixels. As shown in Fig. 1 (a), the most outside circle is the rotating table external aperture. In order to segment only the engine parts, first of all, the effect of the unnecessary aperture should be eliminated. From Fig. 1 (a), the outer ring is not sequential, so the image can be detected by Canny operator, and the complete edge of the engine is obtained. The edge being detected is filled out, as shown in Fig. 1(b). Remove the most outer aperture edge of the binary image, and get the mask image without the unwanted aperture. By multiplying the mask image with the original image, the full engine gray image can be obtained, as shown in Fig. 1 (c). Draw engine gray histogram, as shown in Fig. 1 (d).

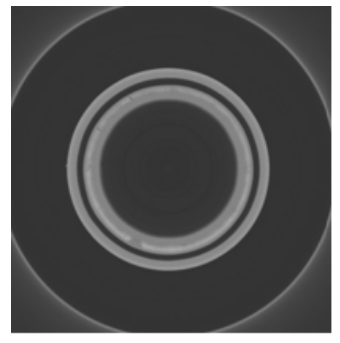

(a) original CT image

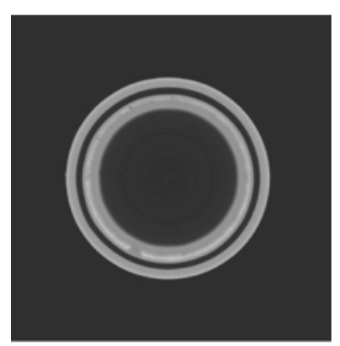

(c) CT image without the aperture

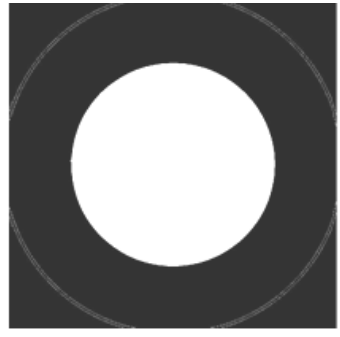

(b) processing of filling out the hole

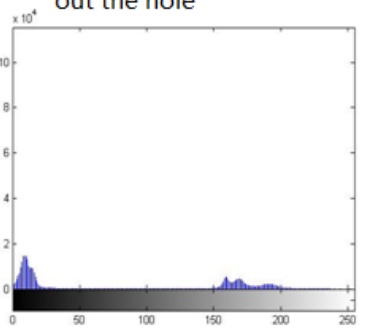

(d) gray histogram of the $\mathrm{CT}$ image without the aperture

Fig. 1: processing of removing the aperture

\subsection{Gray scale analysis and scheme determination}

From Fig. 1 (c) and Fig. 1(d), SRM background and target can be observed clearly. The gray values of the background are distributed among $0 \sim 30$. The engine shell, the insulation layer and the structure of the column structure are mainly distributed among 150 200. The defects of SRM (mainly crack, porosity, and debonding) are mainly distributed in the structure of the propellant, which is a very few of the whole image pixels. Because of the air in the defects, the gray values of the structure are obvious different from the propellant. But due to the reason of equipment caused by the uneven illumination, the gray values of the defects, core hole and propellant are cross each other. Therefore, the defects cannot be accurately segmented using the traditional threshold segmentation method. 
According to the gray distribution of the SRM CT image, the segmentation scheme is determined as Fig. 2:

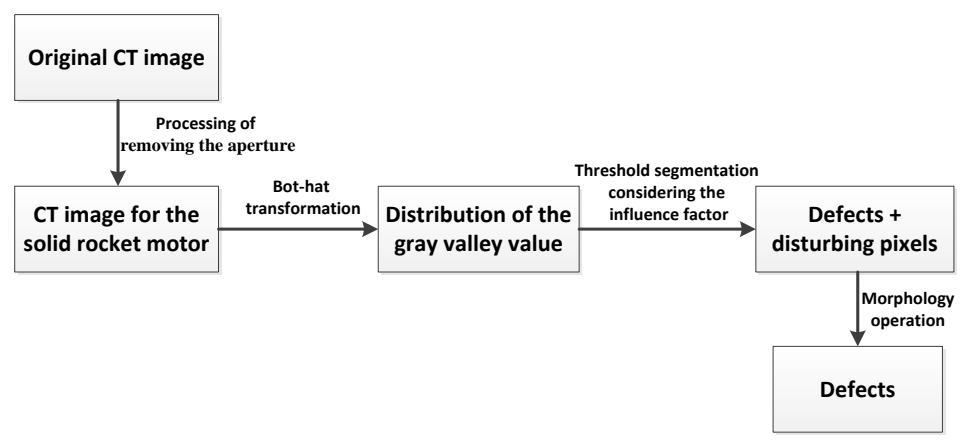

Fig. 2: Scheme for defect detection

\section{Implementation}

\subsection{Bot-hat transformation}

The bot-hat transformation is an operation based on a mathematical morphology, which can enhance the gray valley value in the image, and highlight the extraction of small and dark targets [9]. The definition of the bot-hat transformation is shown in formula (1):

$$
\left.\begin{array}{l}
\text { expansion operation: } \quad(f \oplus b)(x, y)=\max \left\{f\left(x-x^{\prime}, y-y^{\prime}\right)+b\left(x^{\prime}, y^{\prime}\right) \mid\left(x^{\prime}, y^{\prime}\right) \in D_{b}\right\} \\
\text { erosion operation: } \quad(f \Theta b)(x, y)=\min \left\{f\left(x+x^{\prime}, y+y^{\prime}\right)-b\left(x^{\prime}, y^{\prime}\right) \mid\left(x^{\prime}, y^{\prime}\right) \in D_{b}\right\} \\
\text { bot - hat transformation: } \quad B_{\text {bothat }}=f-(f \oplus b) \Theta b
\end{array}\right\}
$$

Wherein, $b$ is a structure element, $f$ is an input image, $D_{b}$ is the definition domain of $b$.

Although the gray value of the propellant decreased, even cross with defect because of the uneven illumination, this part of structure has large area, and the dark areas connect to pieces, so the relationship of propellant pixel points and its neighborhood pixels is significantly different compared with the relationship of defects and its neighborhood pixels. The difference of the characteristics can be extracted by the bot-hat transformation. The result of bot-hat operation of Fig. 1 (c) is shown in Fig. 3 (a). Fig. 3 (b) is the result of the selected region being enlarged 100 times in Fig. 3 (a).

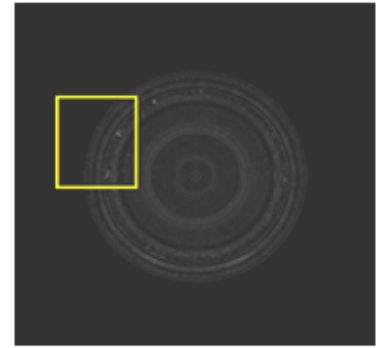

(a) result of bot-hat transformation

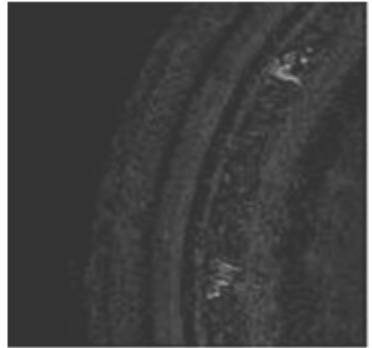

(b) he selected region beinq enlarqed 100

Fig. 3: The result of bot-hat transformation

As can be seen from Fig. 3, after the bot-hat transformation, the defect has been manifested, but the defect contour is not clear. Moreover, in the structure of propellant, there is a large number of interfering pixels which are similar to the feature of the defect pixels. These pixels also have an effect on the defect extraction, which is not conducive to the feature extraction and quantification of the defects, so it also needs to be further processed with the method of threshold segmentation.

\subsection{Threshold segmentation method infusing the influence factor}

The variance of a gray image reflects the uniformity of the image. It is to say that if the differences between the gray values of image pixels are smaller, image will looks more uniform, and the variance 
is small; on the contrary, if the pixel gray level value difference is very big, the image has the obvious contrast, and the variance is big. Observing Fig. 3 (a), in the gray image after the bot-hat there is a very small number of targets in a large dark background. Because the number of interfering pixels is large, the contribution rate of the defect pixels to the variance is small compared with the interfering pixels. Therefore, after the threshold segmentation, we can divide the image into the background and the target defect. The best threshold selected put the background pixels and the interference pixels a class while put the defect pixels which have a small impact factor another class.

Suppose $X$ is an image with the gray level of $L$, and the gray level of $i$ has the number of pixels $N_{i}$. The total number of pixels of the image is $N=\sum_{i=0}^{L-1} N_{i}$. The probability of the pixels belong to the gray level $i$ is $P_{i}=\frac{N_{i}}{N}$. The mean gray level of the whole image is $\mu_{T}=\sum_{i=1}^{L} i P_{i}$. The variance considering the influence factor is calculated by the formula (2).

$$
\sigma^{, 2}=\varepsilon_{0}\left(\mu_{0}-\mu_{T}\right) \wedge 2+\varepsilon_{1}\left(\mu_{1}-\mu_{T}\right)^{\wedge} 2
$$

Among them, $\varepsilon_{0}, \varepsilon_{1}$ is the influence factor of background pixels and target defect pixels respectively, and they have the relationship of $\varepsilon_{0}>\varepsilon_{1}$, and also $\varepsilon_{0}+\varepsilon_{1}=1 . \mu_{0}, \mu_{1}$ is the average gray value of the defect pixels and the background pixels respectively, wherein $\mu_{0}=\sum_{i=0}^{k} i P_{i}, \mu_{1}=\sum_{i=k+1}^{L-1} i P_{i}, k$ is the selected threshold. Draw the trend of variance with the threshold, as shown in Fig. 4.

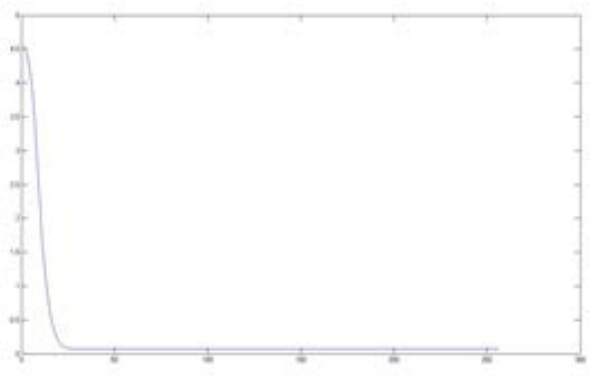

Fig. 4: The trend of variance with the threshold

From Fig. 4, the variance tends to be stable after a certain threshold, and the volatility of the image is small. That is, the gray values of the pixel points exceeded the threshold have the small contribution rate to the whole image. So, the threshold is the best threshold. The threshold can be obtained by difference computation. The optimal threshold of Fig. 3(a) is obtained as $k=30$.

Segment the gray image after bot-hat transformation with the threshold of 30 , and use morphological expansion and erosion operation to eliminate the small, scattered pixels, and transform the defects to binary image. The final segmentation results such as Fig. 5 (a). In order to see the location of defects clearly in the original image, plus the image after top-hat operation and the CT image in which the aperture has been eliminated, and then minus binary defects image as shown in Fig. 5 (b).

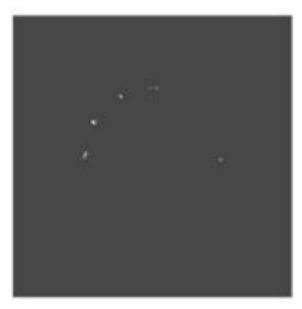

(a) the binary result of the defects

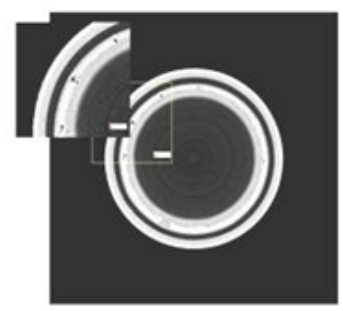

(b) the defects on the background

Fig. 5: The segment result of the defects 
From Fig. 5, it can be seen that the defects had been segmented completely and accurately. In the original context, the distribution and size characteristics of defects can be observed clearly and accurately. The whole contour and the results of binary processing can provide a good basis for the computer to calculate the area, circumference and diameter of the defects in the next step.

\section{Summary}

In this paper, the bot-hat transform and threshold segmentation algorithm are combined. Based on the analysis of the characteristics of engine structure and the gray distribution of the pixels, the bot-hat transformation and the threshold segmentation method considering the impact factor are used to segment the defects. The method can do the bot-hat transform and determine the threshold automatically, having the high intelligent degree. The defects finally got display directly and clearly, which lay a good foundation for a computer to do the intelligent quantitative calculation.

The main conclusions can be drawn as follows:

(1) The proposed method provides an automatic way to detect the defects of SRM.

(2) With the new proposed defects detection method, the spatial distributions, outlines and size characteristics of the defects could be clearly detected.

\section{References}

[1] Yan Liang ,Chi Zhang, Ming Liang, Causal Analysis of Charge Flaws for Solid Rocket Motor and Research on Nondestructive Testing Methods[J], Tactical Missile Technology ,2010, (1) ,73-77.

[2] Rongzhen Liu, Industrial CT Measurement Technology to Solid Rocket Engine [J], Tactical Missile Technology 2008,(5),92-96.

[3] M.Kröning, Th.Jentschl, et al, Non-destructive Testing and Process Control Using X-ray Methods and Radioisotopes,National Association for Applications of Radioisotopes and Radiation in Industry,1998:21-24.

[4] Zhiming Qian, Researches on Image Segmentation Approaches [D], Hunan: National University of Defense Technology, 2010.

[5] Rafael C. Gonzalez, et al, Digital Image Processing Using MATALAB [M], Beijing: Publishing House of Electronics Industry, 2009.

[6] Li chai, 3D-ICT-based Defect-Localization and Feature Extraction Technique for Solid-propellant Rocket Engine [D], Shanxi: North University of China.

[7] Min Zhu, Hongyi Lu, Zhigang Xiao, Binxing Zhang, A kind of automatic segmentation method for solid motor CT image[J], Journal of Solid Rocket Technology, 2008, 31(2), 201-204.

[8] Tao Li, Le Zhang, et al, Analysis and Nondestructive Testing of Defects for Solid Rocket Motor [J], Nondestructive Testing, 2006, 28(10), 541-544.

[9] Shihu Zhu, Function extension and application of morphological top-hat transformation and bottom-hat transformation [J], Computer Engineering and Application, 2001, 47(34), 190-192. 